\title{
Type 2 diabetes mellitus in children: are we 'treating' them right?
}

\author{
Anuar Azriyanti, Zarina Yaakop, Jalaludin Mohammad Yazid, Harun Fatimah \\ From 8th APPES Biennial Scientific Meeting \\ Darwin, Australia. 29 October - 1 November 2014
}

\section{Background}

Incidence of Type 2 diabetes mellitus (T2DM) in children is rising alongside childhood obesity. In children, there is limited therapeutic options and tight blood glucose (BG) control may be challenging.

\section{Aims}

To determine metabolic control and complications in children with T2DM seen in University Malaya Medical Centre (UMMC).

\section{Methods}

Data on children with T2DM referred to and managed in UMMC from 2000 until 2013 were collected. Their body mass index (BMI and blood tests (HbA1c and Lipids) were compared at presentation to their latest clinic appointment. Treatment modalities and duration of follow up were documented. T2DM was dignosed if they had hyperglycaemia (Random BG $>11.8 \mathrm{mmol} / \mathrm{L}$ or fasting BG $>7 \mathrm{mmol} / \mathrm{L}$ or $2 \mathrm{hpp}$ OGTT $>11.1 \mathrm{mmol} / \mathrm{L}$ with low C-Peptide). Hypertension (HPT) if BP> 90th centile for age,sex and height. Dyslipidemia is considered if either triglycerides $>1.7 \mathrm{mmol} / \mathrm{L}$, cholesterol $>5.2 \mathrm{mmol} / \mathrm{L}, \mathrm{HDL}<1.03 \mathrm{mmol} / \mathrm{L}$ or $\mathrm{LDL}>2.50 \mathrm{mmol} / \mathrm{L}$. Non-alcoholic fatty liver disease (NAFLD) was confirmed with ultrasound, diabetic nephropathy (DN) if urine microalbumin $>3.5$ in boys, $>4.5$ in girls and diabetes retinopathy $(D R)$ if reported by opthalmologist.

\section{Results}

A total of 49 children with T2DM were seen, but only 37 had available data for analysis. Their age ranged from 7-17 years old at initial presentation. Forty nine percent $(n=24)$ were boys. The mean duration for follow up was 3.6 years $(0.2-10$ years $)$.
Table 1

\begin{tabular}{|c|c|c|}
\hline & At presentation & At last visit \\
\hline Mean Age: & 11.79 years old $(7-17)$ & 15.3 years old (8.9-22) \\
\hline $\begin{array}{l}\text { Mean } \\
\text { Weight: }\end{array}$ & $61 \mathrm{~kg}$ & $70.4 \mathrm{~kg}$ \\
\hline $\begin{array}{l}\text { Mean BMl } \\
\text { (SDS): }\end{array}$ & $28 \mathrm{kgm}^{2}(+2.62)$ & $28.5 \mathrm{kgm}^{2}(+1.91)$ \\
\hline Mean WC: & $97.0 \mathrm{~cm}$ & $94.3 \mathrm{~cm}$ \\
\hline Mean HbA1c: & $11.2 \%(7.4-16 \%)$ & 9.7\% (5.4-14.4\%) \\
\hline Metformin: & $56 \%(21 / 37)$ & $92 \%(34 / 37)$ \\
\hline Insulin: & $\begin{array}{l}32 \%(12 / 37) \\
\text { ** mainly due to ketosis } \\
\text { at diagnosis }\end{array}$ & $\begin{array}{l}32 \%(12 / 37) \\
{ }^{* *} \text { mainly due to poor BG } \\
\text { control }\end{array}$ \\
\hline Dsylipidemia & $92 \%(22 / 24$ screened $)$ & $87 \%(21 / 24$ screened $)$ \\
\hline Fatty Liver: & $\begin{array}{l}\text { Not screened at } \\
\text { diagnosis }\end{array}$ & $80 \%$ (8/10 screened) \\
\hline Nephropathy: & $\begin{array}{l}\text { Not screened at } \\
\text { diagnosis }\end{array}$ & $24 \%(9 / 37)$ \\
\hline Hypertension & $24 \%(9 / 37)$ & $8 \%(3 / 37)$ \\
\hline Retinopathy: & $\begin{array}{l}\text { Not screened at } \\
\text { diagnosis }\end{array}$ & $\begin{array}{l}1 \text { had early changes of } \\
\text { diabetes retinopathy }\end{array}$ \\
\hline
\end{tabular}

\section{Conclusions}

This study revealed children with Type2DM had poor metabolic control with mean HBAc of $9.7 \%$ and early complications were already seen after 3.6 years of follow up.

Published: 28 April 2015

doi:10.1186/1687-9856-2015-S1-P21

Cite this article as: Azriyanti et al: Type 2 diabetes mellitus in children: are we 'treating' them right? International Journal of Pediatric Endocrinology 2015 2015(Suppl 1):P21. 